\title{
Diffusivities of an Equimolar Methane-Propane Mixture Across the Two-Phase Region by Dynamic Light Scattering
}

\author{
Maximilian Piszko $^{1}$ (D) Cédric Giraudet $^{1}$ (D) Andreas P. Fröba ${ }^{1}$ (D)
}

Received: 7 April 2020 / Accepted: 5 May 2020 / Published online: 23 May 2020

(C) The Author(s) 2020

\begin{abstract}
The present contribution examines the accessibility of diffusivities across the twophase region of an equimolar methane-propane mixture for dynamic light scattering (DLS) experiments. Heterodyne DLS experiments and theoretical calculations of the Rayleigh ratio were performed at 125 different thermodynamic states including the gas, liquid, supercritical, and the two-phase region. The present measurements document that two diffusivities can be determined simultaneously in the liquid state and saturated liquid phase for temperatures and pressures which correspond to densities larger than 1.15 times the critical density. Based on a rigorous assignment of the signals detected in this work, the slow and fast diffusivities could be associated with the Fick and thermal diffusivities. For all other thermodynamic states, a single hydrodynamic mode or signal was obtained experimentally. With the help of theoretical Rayleigh ratios as well as from the general behavior of the diffusivities as a function of temperature and pressure, the signals were identified to be related to the Fick diffusivity in the supercritical state and to a mixed diffusivity in the gas state and the saturated vapor phase. The results are discussed in connection with the behavior of the diffusivities along certain paths in the pressure-temperature projection of the phase diagram of the mixture.
\end{abstract}

Keywords Dynamic light scattering $\cdot$ Equimolar methane-propane mixture $\cdot$ Fick diffusivity $\cdot$ Mixed diffusivity $\cdot$ Phase boundaries $\cdot$ Thermal diffusivity

Cédric Giraudet

cedric.cg.giraudet@fau.de

1 Institute of Advanced Optical Technologies-Thermophysical Properties (AOT-TP), Department of Chemical and Biological Engineering (CBI) and Erlangen Graduate School in Advanced Optical Technologies (SAOT), Friedrich-Alexander-University Erlangen-Nürnberg (FAU), Paul-Gordan-Straße 8, 91052 Erlangen, Germany 


\section{Introduction}

Dynamic light scattering (DLS) is a powerful technique for the determination of transport and other thermophysical properties of pure fluids and fluid mixtures [1, $2]$. The working principle of the technique at macroscopic thermodynamic equilibrium makes it particularly useful for the determination of the Fick and thermal diffusivities close to or across the two-phase region where gradient-based techniques may fail. Experimental and theoretical work [3-5], however, report some limitations of the DLS technique in the near-critical region. Nevertheless, the capabilities and limitations of DLS for the simultaneous determination of Fick and thermal diffusivities across the two-phase region of a binary mixture have not been clearly examined yet.

By DLS, the temporal behavior of scattered light governed by microscopic fluctuations at macroscopic thermodynamic equilibrium is analyzed. In the case of a binary mixture in the hydrodynamic regime, the power spectral density of the fluctuations in temperature and concentration is associated with several transport and other thermophysical properties. Far from a critical point, fluctuations in temperature and in concentration are spatially short-ranged. Their dynamics is only governed by the thermal diffusivity and the Fick diffusion coefficient. Their statics is mainly governed by the specific heat capacity, osmotic compressibility, and optical contrast factors [6,7]. Near a critical point, the mean lifetimes of the fluctuations are governed by the Fick and thermal diffusivities as well as by the thermodiffusion coefficient. The latter arises from the local coupling between heat and mass transfer [3, 7].

In the near-critical region, the interpretation of the signals accessible by DLS is more complicated when only one hydrodynamic mode is resolved. For example, Ackerson and Hanley [5] as well as Fröba et al. [4] could resolve two hydrodynamic modes in their DLS experiments along the critical isochore of methane-ethane mixtures only at a distinct distance away from the critical temperature. Close to the plait critical point, only a single hydrodynamic mode associated with fluctuations in concentration was observed. The limitation regarding the resolution of multiple hydrodynamic modes accessible by DLS is not only restricted to the supercritical state close to the vicinity of the plait critical point. It was also observed close to the consolute critical point of a ternary mixture $[8,9]$, in the saturated liquid phase of a binary mixture at vapor-liquid equilibrium [10-12], and in the liquid state for binary mixtures near their plait critical point [13]. For the last two cases, this limitation was observed close to the region where thermal and Fick diffusivities match.

In this work, we analyzed by DLS the statics and dynamics of fluctuations in temperature and concentration for an equimolar methane-propane mixture over a wide range of thermodynamic states. The range of temperatures and pressures includes the gas, liquid, and supercritical state as well as the liquid and vapor phases under saturation conditions. In addition, the intensities of the fluctuations in temperature and concentration have been theoretically calculated. This is of particular interest for the differentiation of the diffusivities measured by DLS 
when only one hydrodynamic mode is resolved. Besides providing a clear view on the diffusivities accessible by DLS across the two-phase region of an equimolar methane-propane mixture, this work also provides reliable Fick and thermal diffusivity data. Such data might be not only of technical importance [14-16], but also useful for comparison purposes of data derived from molecular dynamics simulations [17].

In the following, the fundamentals of dynamic light scattering from bulk of fluids are given with a special focus on Rayleigh scattering. Afterward, the experimental setup, the data evaluation, and the identification strategy for the interpretation of the signals used in this work are presented. Finally, the results for the Rayleigh ratio and the diffusivities obtained in different thermodynamic states across the two-phase region of an equimolar methane-propane mixture are discussed.

\section{Theoretical Background}

\subsection{Rayleigh Scattering from the Bulk of a Binary Mixture}

The principles of dynamic light scattering (DLS) for the determination of transport and other thermophysical properties are described in detail elsewhere, see, e.g., Refs. $[1,2]$. In the following, only a few aspects of Rayleigh scattering essential for the understanding of this work are presented.

In DLS experiments, the dynamics of microscopic fluctuations with a welldefined wavelength is analyzed. For a binary mixture in macroscopic thermodynamic equilibrium, these fluctuations are associated with fluctuations in temperature and concentration [7, 18]. DLS gives access to their mean lifetimes by calculating the temporal correlation function (CF) of the scattered light intensity. In the case of heterodyne conditions, where much stronger reference light is superimposed to the scattered light coherently, the normalized intensity CF can be written as

$$
\mathrm{g}^{(2)}(\tau)=b_{0}+b_{1} \exp \left(-|\tau| / \tau_{\mathrm{C}, 1}\right)+b_{2} \exp \left(-|\tau| / \tau_{\mathrm{C}, 2}\right) .
$$

In Eq. $1, b_{0}, b_{1}$, and $b_{2}$ are experimental constants determined by the characteristics of the experimental setup as well as by the thermodynamic state. $\tau_{\mathrm{C}, 1}$ and $\tau_{\mathrm{C}, 2}$ represent the mean lifetimes of fluctuations in either temperature or concentration. They are determined by two effective diffusivities $D_{1}$ and $D_{2}$

$$
\tau_{\mathrm{C},\{1,2\}}^{-1}=D_{1,2} q^{2}
$$

and the modulus of the scattering or wave vector

$$
q=4 \pi n \sin \left(\Theta_{\mathrm{S}} / 2\right) / \lambda_{0}
$$

which is accessible by the refractive index of the mixture $n$ at the laser wavelength in vacuo $\lambda_{0}$ and the scattering angle $\Theta_{\mathrm{S}}$. The two effective diffusivities 


$$
D_{1,2}=\frac{1}{2}\left\{\left[a+D_{11}(1+\varepsilon)\right] \mp \sqrt{\left[a+D_{11}(1+\varepsilon)\right]^{2}-4 a D_{11}}\right\},
$$

with

$$
\varepsilon=c^{2}(1-c)^{2} T \frac{S_{\mathrm{T}}^{2}}{c_{\mathrm{p}}}\left(\frac{\partial c}{\partial \mu}\right)^{-1}
$$

are derived from hydrodynamic fluctuations theory [7, 19]. In Eq. 4, $a$ is the thermal diffusivity, $D_{11}$ the Fick diffusivity, and $\varepsilon$ the parameter describing the coupling between heat and mass transfer. In Eq. 5, $c$ is the weight fraction of the heavier component, $T$ the absolute temperature, $S_{\mathrm{T}}$ the Soret coefficient, and $c_{\mathrm{p}}$ the isobaric heat capacity. $(\partial c / \partial \mu)$ is the osmotic compressibility, where $\mu$ is the difference between the chemical potentials of the pure components.

In the liquid state far from the plait critical point, $a$ is at least one order of magnitude larger than $D_{11}$ and $\varepsilon$ tends to 0 . Consequently, the two hydrodynamic modes in Eq. 1 are on different time scales. Furthermore, the fast and slow mode is purely associated with the thermal and Fick diffusivity. In the gas state far from the plait critical point and the dew point line, the thermal and Fick diffusivities have the same order of magnitude and $\varepsilon$ tends again to 0. Typically, $a$ and $D_{11}$ do not differ by more than a factor of 2 , and thus, the two hydrodynamic modes appear on the same time scale. Close to the vicinity of the plait critical point, $D_{11}$ vanishes, $a$ remains nearly constant, $S_{\mathrm{T}}$ and $(\partial c / \partial \mu)$ diverge, and $c_{\mathrm{p}}$ is constant. In the near-critical region, along the critical isochore, there is a temperature $T>T_{\mathrm{c}}$, with $T_{\mathrm{c}}$ the critical temperature, for which the Lewis number $L e=a / D_{11}$ is unity. The smaller the concentration of the solute, the larger the influence of the mode coupling parameter $\varepsilon$, and the closer this temperature is to $T_{\mathrm{c}}[3,19]$. As a criterion, mode coupling is non-negligible until $L e$ is much larger than $\varepsilon$. In general, the divergence of $\varepsilon$ approaching the critical temperature is weaker than the divergence of $L e$ [3].

Whether both modes can be resolved is mainly restricted by the amplitudes of the signals associated with the fluctuations in temperature and concentration. They cannot be determined experimentally in an absolute way due to unknown experimental constants. In contrast, the Rayleigh ratio representing the ratio of signal amplitudes $b_{\mathrm{c}}$ and $b_{\mathrm{t}}$ related to the fluctuations in concentration and temperature, i.e., either $b_{1}$ or $b_{2}$, is accessible experimentally. The Rayleigh ratio is defined by [7, 19]

$$
\mathfrak{R}=\frac{b_{\mathrm{c}}}{b_{\mathrm{t}}}=\frac{c_{\mathrm{p}}}{T}\left(\frac{\partial c}{\partial \mu}\right) \frac{\left(\frac{\partial n}{\partial c}\right)^{2}}{\left(\frac{\partial n}{\partial T}\right)^{2}} .
$$

For Rayleigh ratios larger than one, the signal in the CF with the larger amplitude is associated with fluctuations in concentration and the signal with the smaller amplitude is associated with fluctuations in temperature. When $\mathfrak{R} \ll 1$ or $\Re \gg 1$, a single exponential is observed. The closer $\mathfrak{R}$ or $b_{1} / b_{2}$ is to unity, the larger the deviation of $L e$ or $D_{2} / D_{1}$ from unity must be to detect experimentally two hydrodynamic modes. 
When only one hydrodynamic mode is resolvable, knowledge about the Rayleigh ratio gives an indication about the kind of fluctuations observed. This is, however, restricted to the case where $L e \gg 1$. When $L e$ and $\Re$ are close to unity, the single hydrodynamic mode reflects within combined uncertainty the dynamics of both temperature and concentration fluctuations $[10,12]$.

\subsection{Refractive Index and Optical Contrast Factors}

The refractive index of the binary mixture can be estimated using the Lorentz-Lorenz equation [20] combined with a linear mixing rule [21]

$$
\frac{n^{2}-1}{n^{2}+2}=\frac{N_{\mathrm{a}}}{3 \varepsilon_{0}} \rho\left(\frac{c \alpha_{\mathrm{e}, 1}}{M_{1}}+\frac{(1-c) \alpha_{\mathrm{e}, 2}}{M_{2}}\right) .
$$

In Eq. 7, $N_{\mathrm{a}}$ is the Avogadro constant, $\varepsilon_{\mathrm{o}}$ the permittivity of free space, $\rho$ the density of the mixture. $\alpha_{\mathrm{e}, \mathrm{i}}$, and $M_{\mathrm{i}}$ are the electronic polarizability and molar mass of the component $i$, respectively.

The optical contrast factors $(\partial n / \partial T)$ and $(\partial n / \partial c)$ can be calculated using the following well-established analytical expressions [21-23]

$$
\frac{\partial n}{\partial T}=\frac{\left(n^{2}-1\right)\left(n^{2}+2\right)}{6 n} \times \frac{1}{\rho} \frac{\partial \rho}{\partial T}
$$

and

$$
\frac{\partial n}{\partial c}=\frac{\left(n^{2}-1\right)\left(n^{2}+2\right)}{6 n}\left(\frac{1}{\rho} \frac{\partial \rho}{\partial c}+\frac{\frac{\alpha_{\mathrm{e}, 1}}{M_{1}}-\frac{\alpha_{\mathrm{e}, 2}}{M_{2}}}{\frac{c \alpha_{\mathrm{e}, 1}}{M_{1}}+\frac{(1-c) \alpha_{\mathrm{e}, 2}}{M_{2}}}\right) .
$$

\section{Experimental Section}

\subsection{Materials}

The equimolar methane-propane mixture investigated in this work was provided by Linde AG in a gas bottle with an initial pressure of 1.284 MPa. The expanded uncertainty $(k=2)$ in the mixture composition stated by the supplier is $1 \mathrm{~mol} \%$. The purities of the pure components methane $\left(\mathrm{CH}_{4}, \mathrm{CAS} \# 74-82-8\right)$ and propane $\left(\mathrm{C}_{3} \mathrm{H}_{8}\right.$, CAS \# 74-98-6) are specified as (99.995 and 99.95) mol\%. The sample was used without further purification.

\subsection{Experimental Setup and Conditions}

In the following, the sample preparation, the experimental setup, the experimental protocol, and the measurement conditions are briefly described. More details about 
the experimental setup including the optical arrangement, the sample cell, the pressure and temperature control can be found in Refs. [13, 24].

Before filling the pressure system, including the sample cell, an oil-sealed vacuum pump was used to lower the pressure down to about 0.4 Pa. Thereafter, the whole system was filled with the sample at room temperature to a pressure of $0.2 \mathrm{MPa}$. This evacuation and pre-filling procedure was repeated three times to ensure high purity of the sample investigated. After filling the mixture, it was processed close to the state of interest by means of a lubricant-free gas compressor and a spindle press. For a fine adjustment of the pressure in the sample cell as well as for pressure stabilization during the experiments, resistance heating of the expansion bellows attached to the sample cell was applied [13]. Also for the measurement cell, resistance heating was applied for temperature control. In the present investigations, the temperature and pressure stabilities in the sample cell were on average $\pm 0.3 \mathrm{mK}$ and $\pm 0.002 \mathrm{MPa}$. Temperatures and pressures were measured with the help of calibrated Pt100 resistance probes and pressure transducers with an expanded uncertainty $(k=2)$ of $20 \mathrm{mK}$ and $0.008 \mathrm{MPa}$.

The optical and electronic arrangement of the experimental setup used in this work consists of a continuous-wave laser, photomultiplier tubes (PMTs), and an auto-collimation system. The laser is operated at $\lambda_{0}=532.1 \mathrm{~nm}$ and focused into the sample cell via a combination of mirrors and a lens. The optical system is aligned so that the irradiating focused beam and the axis of observation, which is given by two circular apertures with diameters of $1 \mathrm{~mm}$ distanced by about $4 \mathrm{~m}$, intersect in the middle of the sample cell. By applying a well-defined incident angle $\Theta_{\mathrm{I}}$, which is the angle between the direction for the detection of the scattered light and the main beam, the scattering geometry is adjusted. The incident angle was measured by autocollimation with an estimated expanded uncertainty $(k=2)$ of $0.01^{\circ}$. An additional reference beam can be superimposed on the scattered light to ensure a sufficiently high degree of heterodyning. For this, part of the incident beam is reflected by a beam splitter and its intensity is controlled by a combination of a lambda half-wave plate and a polarization beam splitter. The same combination is also used for the control of the intensity of the main beam. Two PMTs operated in a pseudo-crosscorrelation scheme are used to detect the time-dependent scattered light intensity.

For each thermodynamic state, 2 to 6 individual measurements were performed at angles of incidence $\Theta_{\mathrm{I}}$ between $(2.3 \text { and } 4.5)^{\circ}$, whereby the main beam was directed from each side with respect to the detection direction. The range of thermodynamic states investigated in this work does not include the vicinity of the plait critical point where the sample becomes opalescent and gravity induces density gradients [25]. For that reason, a backscattering geometry was not needed and the measurements could always be performed in the middle of the sample cell. The pulses of the PMTs are discriminated, amplified, and fed simultaneously to a linear-tau as well as a multi-tau correlator calculating the normalized pseudo-cross-intensity CF. For all measurements, laser-heating effects could be avoided by reducing the incident laser power to a minimum.

As shown in Fig. 1, the binary mixture was studied at 125 different thermodynamic state points including the gas ( $\mathrm{G}$, diamonds), supercritical (SC, circles), liquid (L, squares) states, as well as the two-phase region (2P, triangles). The two-phase 
Fig. 1 Two-phase boundary line (solid blue line) and plait critical point (closed red circle) of the equimolar methane-propane mixture calculated with the GERG-2008 EoS [26]. The black dotted and/or dashed lines denote selected isochores discussed in this work. The different symbols indicate all state points investigated in this work (Color figure online)

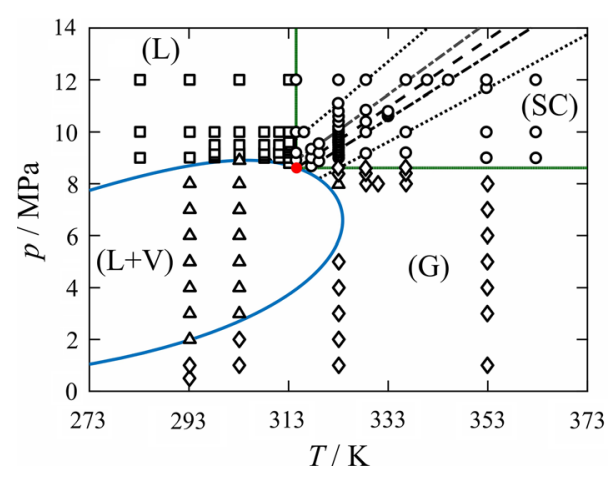

boundary line of the mixture calculated using the GERG-2008 equation of state (EoS) [26] as implemented in the REFPROP database version 10 [27] is represented in Fig. 1 by the solid blue line. The closed red circle represents the plait critical point which is estimated to be located at $T_{\mathrm{c}}=314.06 \mathrm{~K}$ and $p_{\mathrm{c}}=8.65 \mathrm{MPa}$. $p_{\mathrm{c}}$ is the critical pressure. The dashed, dotted-dashed, and dotted lines in Fig. 1 represent selected theoretical isochores corresponding to the critical isochore $\left(\rho_{\mathrm{c}}=239.61 \mathrm{~kg} \cdot \mathrm{m}^{-3}\right)$, $\rho=\rho_{\mathrm{c}} \pm 10 \mathrm{~kg} \cdot \mathrm{m}^{-3}$, and $\rho=\rho_{\mathrm{c}} \pm 33 \mathrm{~kg} \cdot \mathrm{m}^{-3}$, respectively. While the GERG-2008 EoS [26] represents the standard for predicting the phase behavior of natural gases, it is important to note that for an equimolar methane-propane mixture, deviations of $10 \mathrm{~K}$ for $T_{\mathrm{c}}$ and $0.3 \mathrm{MPa}$ for $p_{\mathrm{c}}$ can be found between the predictions from the GERG-2008 EoS [26] and literature data [28].

\subsection{Data Evaluation}

For an accurate analysis of the mean lifetimes of temperature and concentration fluctuations from the measured intensity $\mathrm{CFs}$, an additional first- or second-order polynomial was added to the theoretical fit model given by Eq. 1 if necessary. The additional term accounts for disturbances observed especially in the near-critical region. Although their physical origin could not be identified, an influence on the measured diffusivities could be neglected. All CFs were fitted using the nonlinear Levenberg-Marquardt algorithm.

The upper part of Figs. 2 show two experimental CFs calculated by the linear-tau correlator. The CF illustrated in the upper part of Fig. 2a was obtained at $T=283.17 \mathrm{~K}, p=12.000 \mathrm{MPa}$, and $\Theta_{\mathrm{I}}=4.00^{\circ}$. Here, two hydrodynamic modes can be resolved, and the fit model proposed by Eq. 1 and depicted by the solid red line describes well the experimental CF. In this work, we always associate the slow mode with the relaxation time $\tau_{\mathrm{C}, 1}$, and the fast mode with the relaxation time $\tau_{\mathrm{C}, 2}$. The fast mode indicated by the blue dotted line has a smaller amplitude than the slow mode indicated by the green dashed line. In contrast, for the CF shown in the upper part of Fig. $2 \mathrm{~b}$ obtained at $T=362.61 \mathrm{~K}, p=10.000 \mathrm{MPa}$, and $\Theta_{\mathrm{I}}=4.00^{\circ}$, only a single exponential could be resolved. This finding could be confirmed by the residual plot which is free of any systematics, see lower part of Fig. $2 b$. 
(a)

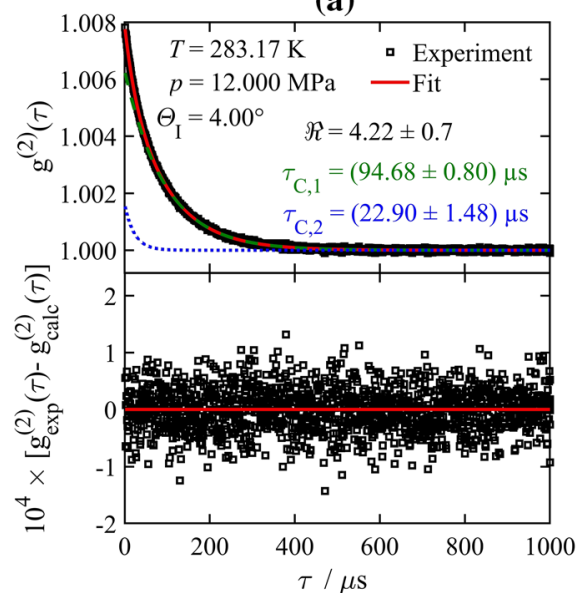

(b)

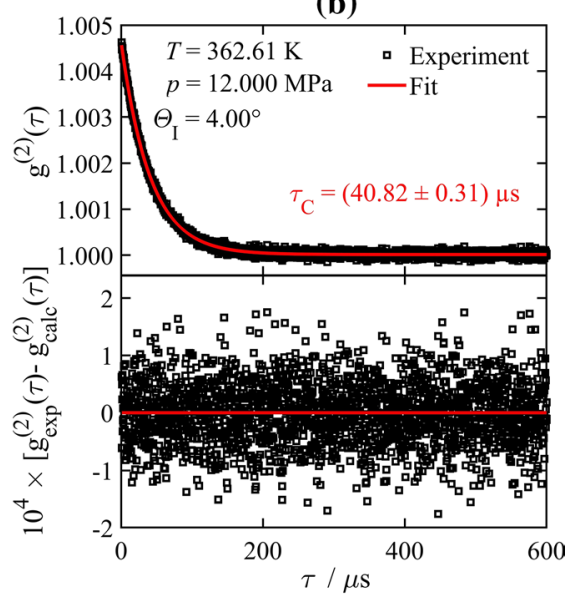

Fig. 2 Fit to CFs (upper parts) and residuals from the fit to CFs (lower parts) recorded by a linear-tau correlator for an equimolar methane-propane mixture at $T=283.17 \mathrm{~K}, p=12.000 \mathrm{MPa}$, and $\Theta_{\mathrm{I}}=4.00^{\circ}$ (a) as well as at $T=362.61 \mathrm{~K}, p=12.000 \mathrm{MPa}$, and $\Theta_{\mathrm{I}}=4.00^{\circ}(\mathbf{b})$ (Color figure online)

The diffusivities were derived from Eq. 2 using the mean lifetimes of the fluctuations $\tau_{\mathrm{C}, 1}$ and $\tau_{\mathrm{C}, 2}$ and the modulus of the wave vector $q$. The latter was determined by Eq. 3 and the law of Snell-Descartes using the angle of incidence $\Theta_{\mathrm{I}}$, and the refractive index of the mixture estimated via Eq. 7. For the present range of incident angles, an estimation of $n$ within $\pm 50 \%$, which is about three orders of magnitude larger than the typical error in the calculated $n$ values, will introduce errors in $q$, and thus, $D_{1,2}$ of $0.03 \%$ and $0.06 \%$, which are clearly below those related to the uncertainty in $\Theta_{\mathrm{I}}$.

Finally, the diffusivity data reported for a defined thermodynamic state represent weighted averages of the individual measurements recorded with two types of correlators at different scattering geometries. The weighting scheme is based on the inverse relative uncertainty of individual measurements determined by uncertainty propagation of the statistical uncertainty from the fit as well as the uncertainty in the incident angle [10, 13]. The relative uncertainties in the diffusivities represent weighted double standard deviations implying a confidence level of $95 \%$ [10]. A similar procedure was also used for the determination of the experimental Rayleigh ratio and its uncertainty. The temperatures and pressures reported are mean values recorded during the complete measurement series.

\subsection{Rayleigh Ratio Calculations}

The Rayleigh ratio was calculated by Eqs. 6-9 using polarizabilities from the literature and mixture data for the density, specific heat capacity, and chemical potential of the components estimated by the GERG-2008 EoS [26] or the volume translated Peng-Robinson (VT-PR) EoS [29], as implemented in the REFPROP database version 10 [27] using experimental temperatures and pressures. The polarizability of 
propane was fixed to the experimental value of the monomer proposed by the NIST Computational Chemistry Comparison and Benchmark Database [30] and the polarizability of methane to the effective one proposed by Giraudet et al. [23] In the case of propane, the lack of experimental refractive index data at temperatures and pressures relevant for this work did not allow to determine a suitable effective polarizability. The uncertainty reported for the GERG-2008 EoS [26] in density is between (0.03 and 0.3) \% [26]. Richter and McLinden [31] have shown, however, that deviations up to more than $1 \%$ between the values predicted by the GERG-2008 EoS [26] and recent density measurements can be found for methane-propane mixtures in the gas state. The osmotic compressibility and the partial derivatives of the density were calculated by describing the mass fraction or temperature dependencies of $\mu$ and $\rho$ around the experimental temperatures and composition by a first- or second-order polynomial.

\subsection{Strategy for Signal Identification and Interpretation}

In the following, a strategy allowing the identification and interpretation of the signals recorded in the $\mathrm{CF}$ is presented. When two hydrodynamic modes are resolvable in the experimental CF, the Rayleigh ratio $\Re$ and the two diffusivities $D_{1}$ and $D_{2}$ are determined. As already mentioned, $D_{1}$ and $D_{2}$ are the diffusivities derived from the slow and fast mode. The interpretation of the two modes, i.e., whether they can be associated either with fluctuations in concentration or with fluctuations in temperature, is carried out by theoretical analysis of the Rayleigh ratio. If $\mathfrak{R}>1$, the mode with the larger signal amplitude is associated with fluctuations in concentration, the mode with the smaller signal amplitude with fluctuations in temperature. The situation with respect to the signal amplitude is inverted if $\mathfrak{R}<1$. The identification of the two diffusivities $D_{1}$ and $D_{2}$ is further supported via an analysis of their behavior as a function of temperature and pressure. In the absence of a coupling between heat and mass transfer, $D_{1}$ and $D_{2}$ are associated with the pure Fick or thermal diffusivities. If such a mode coupling exists, however, $D_{1}$ and $D_{2}$ are associated with neither $D_{11}$ nor $a$, but with two effective diffusivities. When only one hydrodynamic mode is resolved in the $\mathrm{CF}$, the identification of the mode and the diffusivity $D$ is also performed based on the theoretically estimated Rayleigh ratio and the general behavior of $D$. If $\Re \gg 1$, the single mode is associated with fluctuations in concentration and $D$ is associated with $D_{11}$ in the absence of mode coupling or with the effective diffusivity $D_{1}$ in the presence of mode coupling. If $\Re \ll 1$, the single mode is associated with fluctuations in temperature and $D$ is associated with $a$ in the absence of mode coupling or the effective diffusivity $D_{2}$ in the presence of mode coupling. If $\mathfrak{R}$ is close to unity, the single signal observable in the CF is governed by both concentration and temperature fluctuations, and thus, it can be related only to a mixed diffusivity $D_{\mathrm{m}}$. In case that the Lewis number $L e$ is also close to unity and no mode coupling is present, $D_{\mathrm{m}}$ matches with both $a$ and $D_{11}$. Of course, this interpretation is connected to an extended uncertainty which depends on the distance of Le from unity and which might rapidly increase. 
In the following, Fick, thermal, and mixed diffusivities are depicted by solid, open, and half-filled symbols, respectively. The suggested strategy for the interpretation of diffusivities accessible by DLS requires accurate information about the Rayleigh ratio. If this is lacking, enormous errors result from the interpretation, although the statistical uncertainty of the DLS experiments is very small.

\section{Results and Discussion}

For the equimolar methane-propane mixture, DLS experiments were conducted at 125 different thermodynamic states. As shown in Fig. 1, the study includes 36 state points in the liquid (L) state, 53 in the supercritical (SC) state, 21 in the gas (G) state, and 15 in the two-phase (2P) region. For the latter, both the saturated liquid $(2 \mathrm{P}-\mathrm{L})$ and saturated vapor $(2 \mathrm{P}-\mathrm{V})$ phases were investigated whenever it was possible. Here, the composition of the mixture in each phase varies with the pressure and the temperature. In the following, diffusivity results accessible in different regions of the phase diagram of the equimolar methane-propane mixture are presented. Thereafter, the results for the diffusivities and Rayleigh ratios are presented along selected isobars, isotherms, and isochores across the two-phase region of the mixture.

\subsection{Diffusivities and Rayleigh Ratios}

For all thermodynamic states, experimental diffusivities and Rayleigh ratios obtained by DLS are summarized together with their associated expanded relative uncertainties $(k=2)$ in Table 1 . For clarity reasons, only selected results for the diffusivities and Rayleigh ratio are presented in the following figures. The error bars represent the experimental expanded uncertainties $(k=2)$. The diffusivities vary by 3 orders of magnitude and exhibit expanded uncertainties $(k=2)$ between $(0.2$ and 84.8) $\%$. On average, the expanded uncertainty is $6.9 \%$. Diffusivity data with a relatively high uncertainty are always associated with the fast mode when two hydrodynamic modes can be resolved. This is because the amplitude of the signal associated with the fast mode was always found smaller than the amplitude in the signal associated with the slow mode.

In this work, two hydrodynamic modes could be resolved for temperatures and pressures which correspond to a relative density $\rho / \rho_{\mathrm{c}}$ larger than 1.15 . The experimental Rayleigh ratio varies by nearly 2 orders of magnitude and exhibits expanded uncertainties $(k=2)$ between (3.9 and 77.5) \%. The average expanded uncertainty $(k=2)$ is $27.1 \%$. Here, large uncertainty for $\mathfrak{R}$ can be found in the vicinity of the two-phase boundary line.

\subsection{Diffusivities in the Liquid and Supercritical States Along Isobars}

The experimental results obtained for the diffusivities $D_{1}$ and $D_{2}$ along three isobars crossing the liquid and supercritical states of the mixture are represented in Fig. 3. The diffusivities obtained over the temperature range from (283 to 363) K 


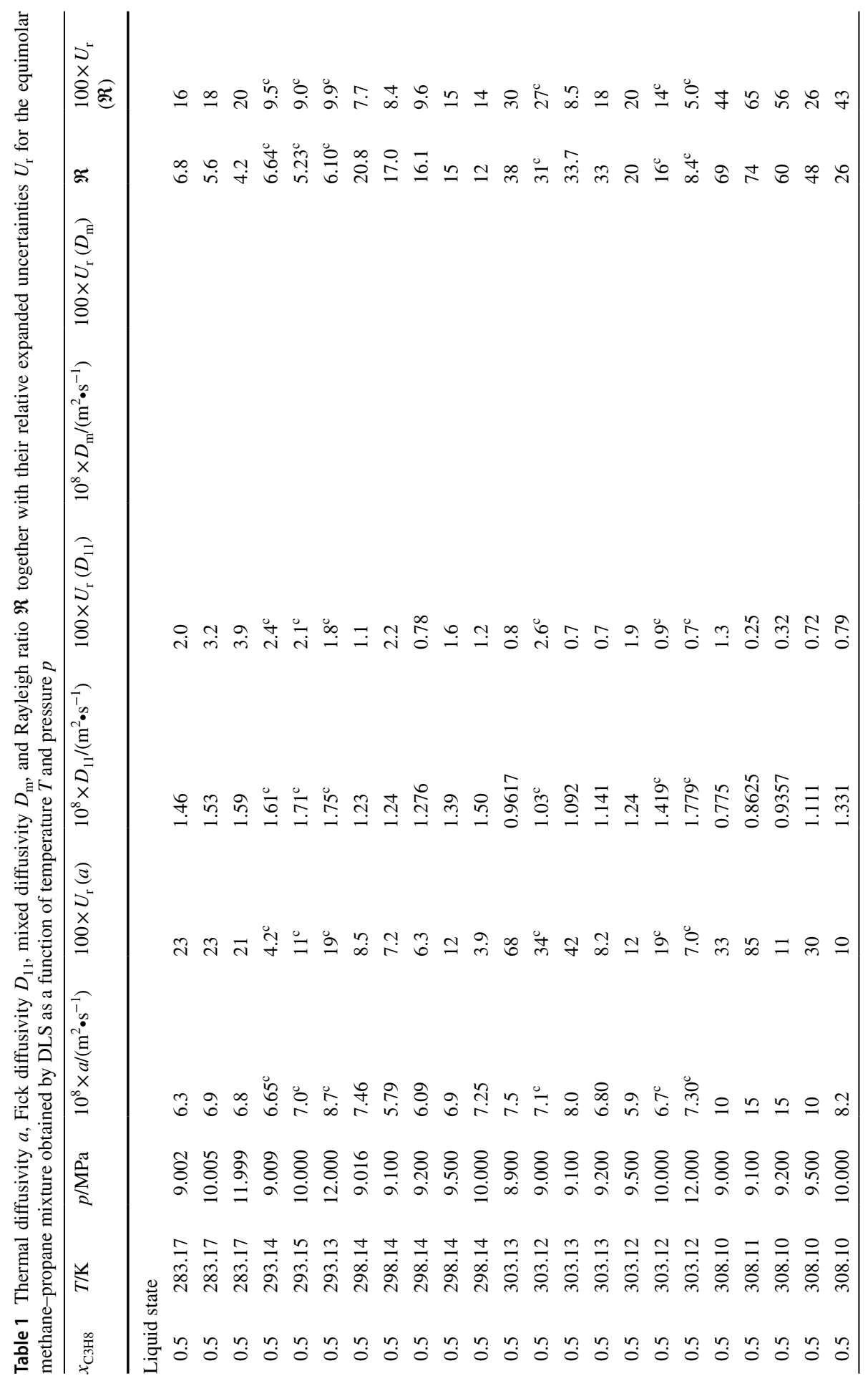




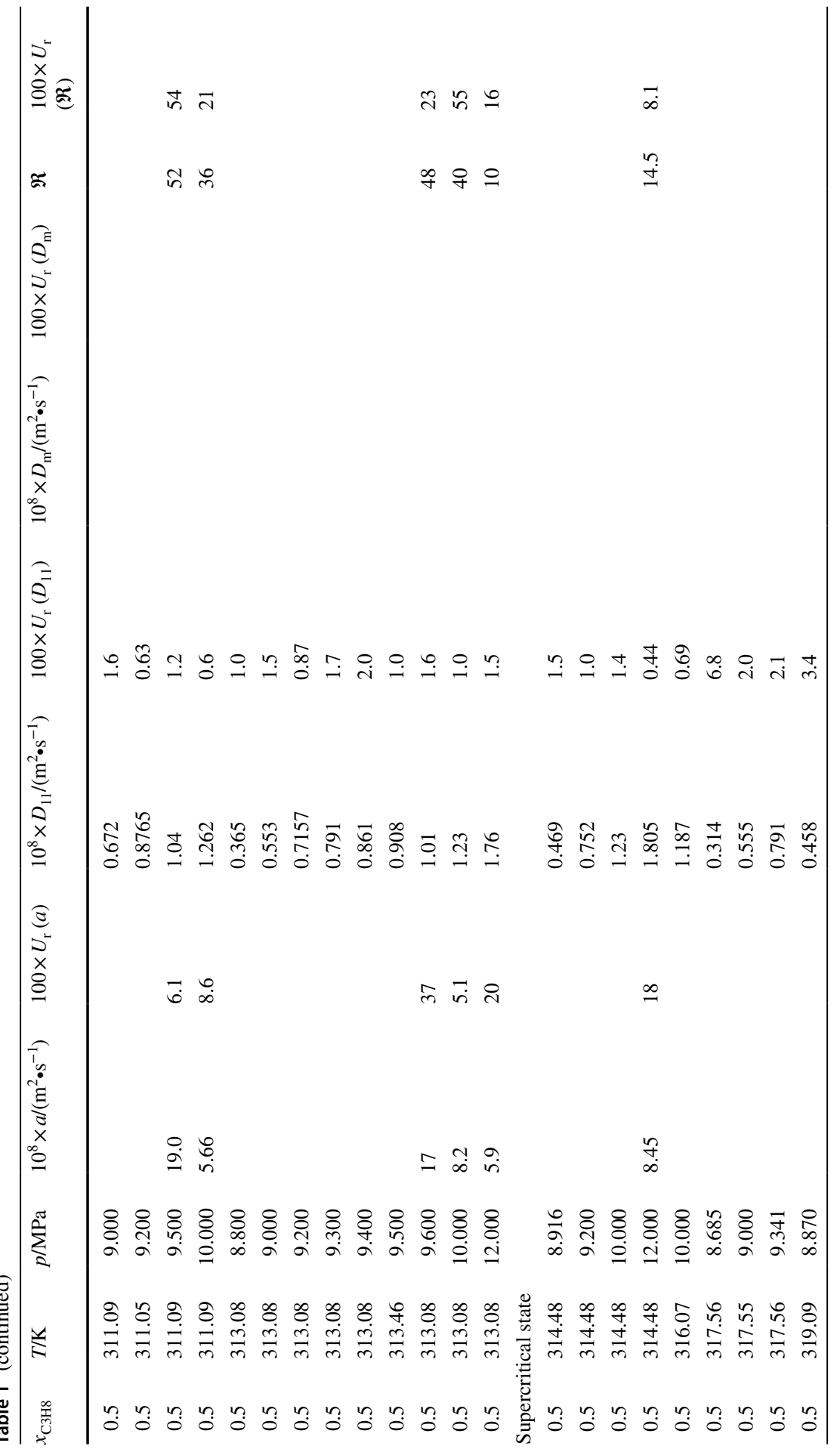




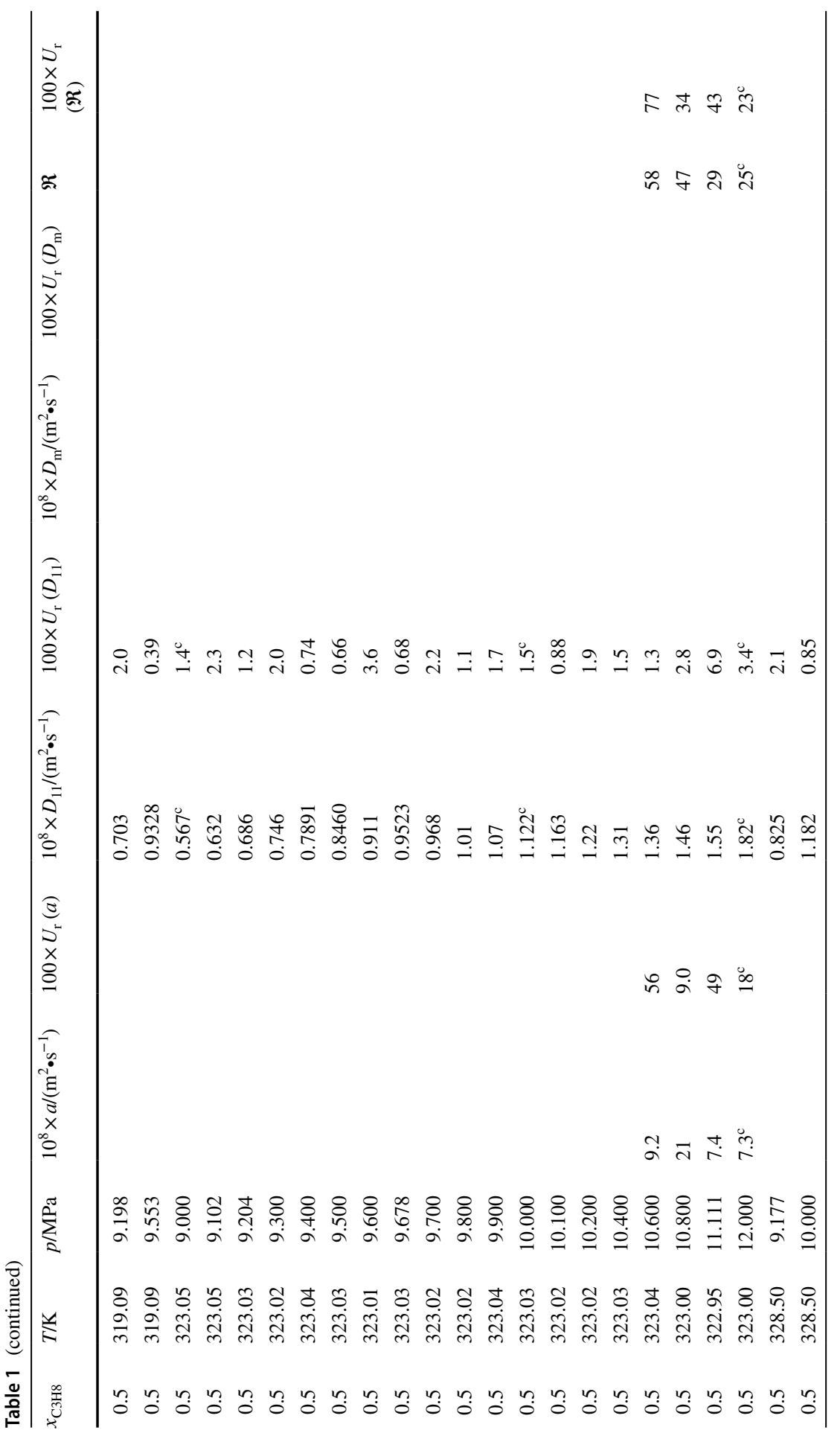




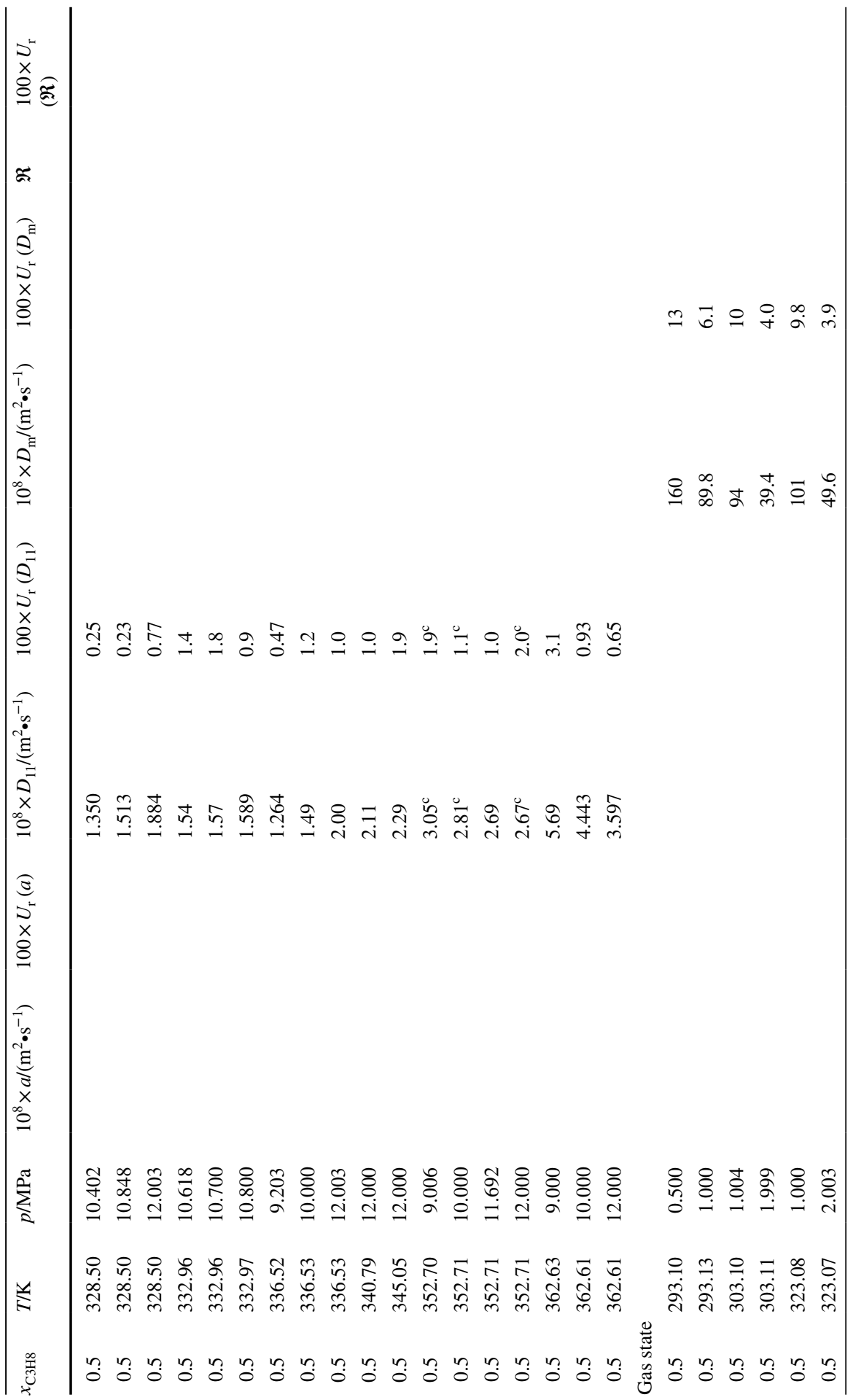




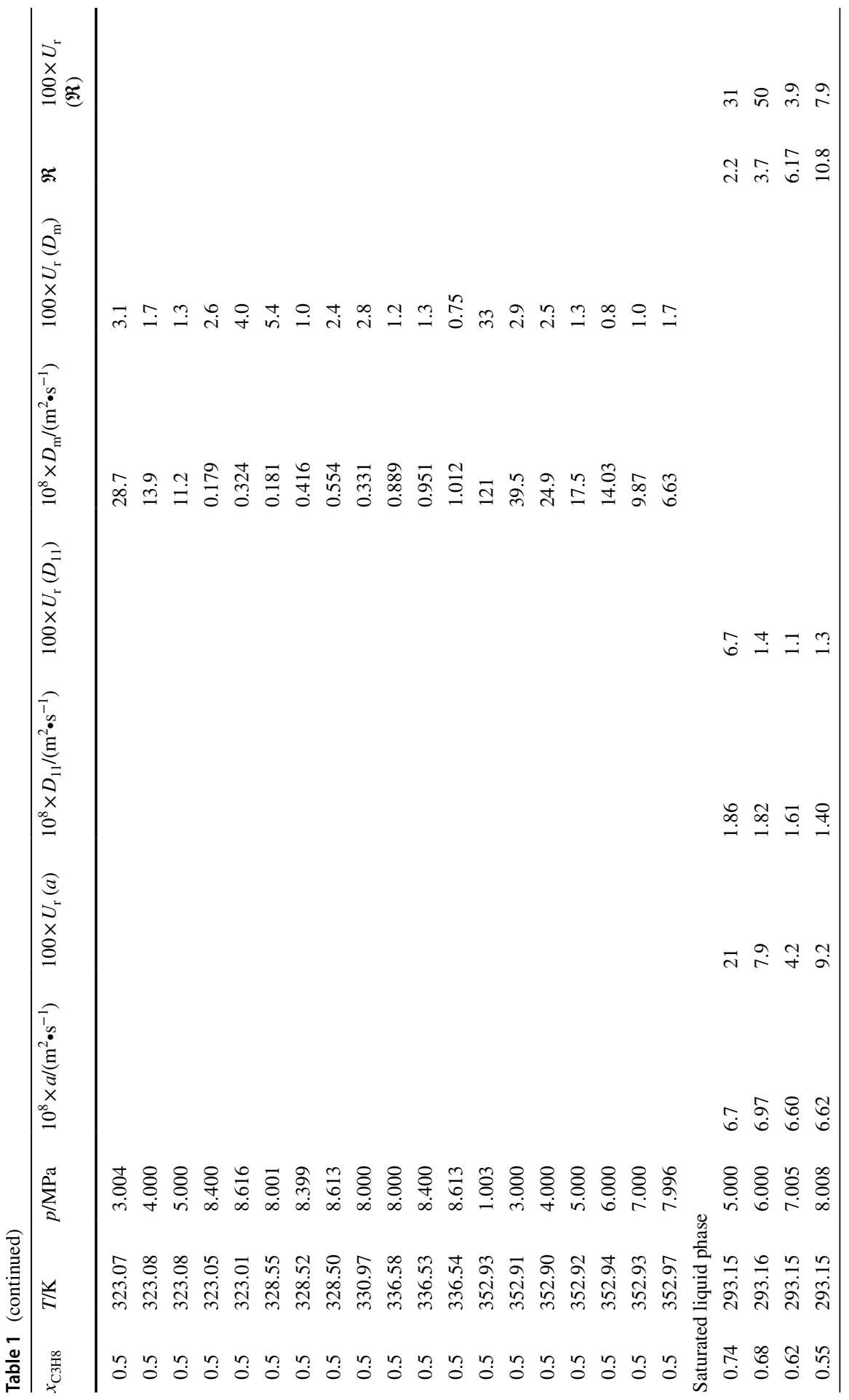




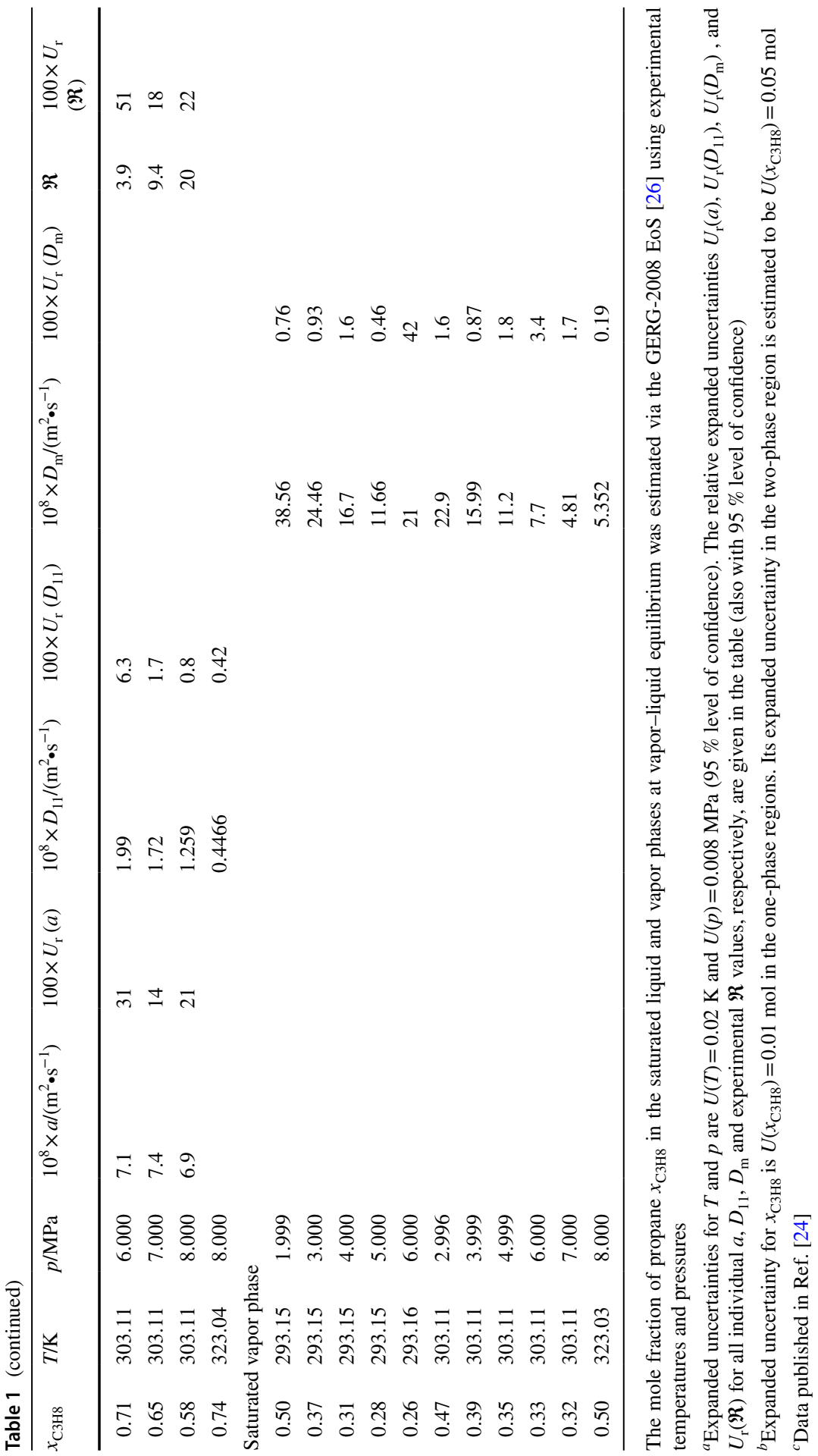


Fig. 3 Slow or Fick diffusivity (upper part) and fast or thermal diffusivity (lower part) as a function of temperature along three isobars

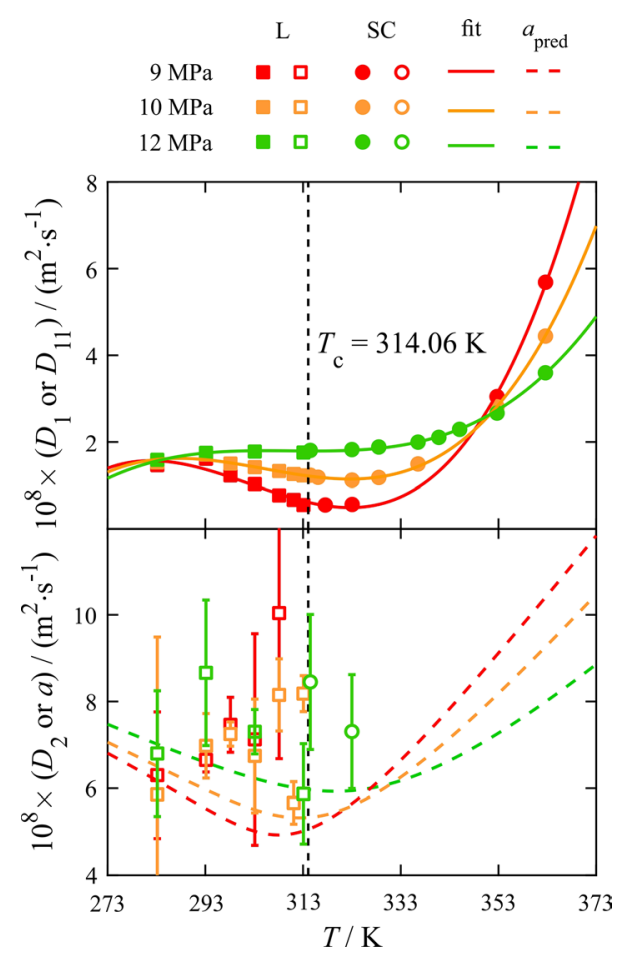

at pressures of $(9,10$, and 12) MPa are depicted by red, orange, and green symbols, respectively. As it can be seen in Fig. 3, two diffusivities $D_{1}$ and $D_{2}$ were mainly resolved in the liquid state. Here, the ratio between the fast diffusivity $D_{2}$ and the slow diffusivity $D_{1}$ is varying between 4 and 13 . The closer the density to $\rho_{\mathrm{c}}$, the larger the ratio. The behavior of $D_{1}$ which can be interpreted with the Fick diffusion coefficient $D_{11}$, as it will be shown later, is represented in the upper part of Fig. 3 . The behavior of $D_{2}$ which can be interpreted with the thermal diffusivity $a$, which is also shown later, as a function of temperature is illustrated in the lower part of Fig. 3. The dashed lines in the lower part of Fig. 3 represent the thermal diffusivity calculated by REFPROP [27], which will be discussed later on.

For most of the temperatures and pressures corresponding to the supercritical state, only one hydrodynamic mode was resolved. For the three isobars, the behavior of the resulting diffusivity follows that one of $D_{1}$. Hence, we can assume that the single hydrodynamic mode resolved in the supercritical state is also associated with $D_{11}$. As indicated by the solid lines in the upper part of Fig. 3, the temperature dependence of $D_{11}$ across the liquid and supercritical states can be well represented by a third-order polynomial.

For the three isobars shown in Fig. 3, the theoretical and experimental Rayleigh ratios as a function of temperature are illustrated in Fig. 4. The experimental data are indicated by the symbols and the solid and dashed lines represent $\mathfrak{R}$ values calculated via Eq. 6 using properties predicted by the GERG-2008 EoS [26] and the VT-PR EoS [29]. Far from the plait critical point, theoretical and 
Fig. 4 Experimental (symbols) and theoretical (lines) Rayleigh ratios as a function of temperature at different pressures. The solid lines illustrate the results obtained with the GERG-2008 EoS [26] and the dashed lines the predictions using the VT-PR EoS [29]

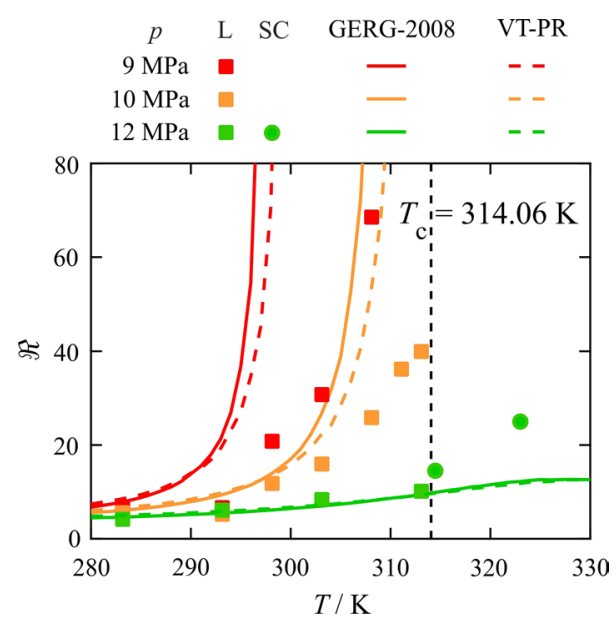

experimental $\mathfrak{R}$ values are in excellent agreement. For example, at $T=293.13 \mathrm{~K}$ and $p=12.000 \mathrm{MPa}$, the experimental Rayleigh ratio is $(6.1 \pm 0.6)$ and the values predicted by the GERG-2008 EoS [26] and the VT-PR EoS [29] are 5.9 and 5.8. In general, Rayleigh ratios estimated with the VT-PR EoS [29] are closer to the experimental values. Approaching the plait critical point, large discrepancies between experimental and theoretical Rayleigh ratios are observable. Here, it could be verified that this discrepancy is not related to an under- or overestimation of the polarizabilities of methane and propane. It should be noted that the critical temperature estimated with the VT-PR EoS [29] is $7.05 \mathrm{~K}$ larger than the value predicted by the GERG-2008 EoS [26]. Yet, it is unlikely that the specification of $T_{\mathrm{c}}$ is responsible for the observed deviations between experiments and calculations. The more likely reason for the discrepancies is a misevaluation of the osmotic compressibility in the near-critical region [32].

For each thermodynamic state where two modes were resolved along the isobars, experiments and theoretical calculations agree in connection with the mode identification. The fast mode and the slow mode are associated with fluctuations in temperature and in concentration. In the following, despite the general better agreement for the Rayleigh ratio with the VT-PR EoS [29] in the liquid state, we only used the GERG-2008 EoS [26] because it better predicts the density of methane-propane mixtures over the complete range of temperatures and pressures investigated in this work [26, 29, 31]. Figure 5 shows the behavior of $\mathfrak{R}$ around the two-phase region of the equimolar methane-propane mixture predicted with the GERG-2008 EoS [26]. Calculations close to the plait critical point, however, could not be performed. Over the complete range of temperatures investigated in the one-phase region for $p>p_{\mathrm{c}}$, fluctuations in concentration are dominating the scattered light intensity. This confirms that the single mode detected in the supercritical state can also be associated with fluctuations in concentration. This is particularly true for temperatures and pressures smaller than $355 \mathrm{~K}$ and $12 \mathrm{MPa}$, where the amplitude in the signal associated with fluctuations in concentration is at least 1 order of magnitude larger than that one associated with fluctuations in temperature. 
Fig. 5 Isolines of the Rayleigh ratio predicted using the GERG2008 EoS [26] in the liquid and supercritical states

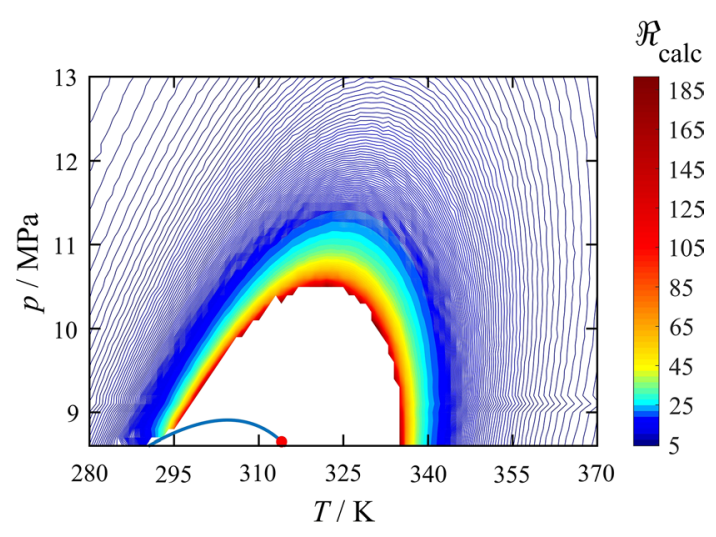

Our interest is also directed to the question whether the diffusivities $D_{1}$ and $D_{2}$ can be associated with either pure thermal or Fick diffusivities as it was claimed before. This is qualitatively confirmed by the reasonably good agreement between experimental $D_{2}$ data and data for the thermal diffusivity $a$ predicted by the REFPROP Database [27] and depicted by dashed lines in the lower part of Fig. 3. For this study, the lack of knowledge about the Soret coefficient and the inability of the GERG-2008 EoS [26] to obtain osmotic compressibility data in the near-critical region precluded the calculation of the mode coupling parameter. Nevertheless, a possible influence of a coupling between heat and mass transfer can be qualitatively evaluated by analyzing the crossover between the two diffusivities, i.e., by determining the temperatures for which the Lewis number is unity for the three isobars investigated in this work. At temperatures and pressures smaller than $355 \mathrm{~K}$ and $12 \mathrm{MPa}$, the amplitude of the signal associated with fluctuations in concentration is much larger than that one associated with fluctuations in temperature, and $L \mathrm{e}$ is larger than 2. For a pressure of $(9,10$, and 12$) \mathrm{MPa}, L e$ is expected to be equal to unity at a temperature of about $(65,73$, and 87$) \mathrm{K}$ away from $T_{\mathrm{c}}$, respectively. As mentioned in Sect. 2, a mode coupling has to be considered only close to the vicinity of the crossover between $a$ and $D_{11}$, and thus, can be neglected over the complete temperature and pressure range investigated in this work. This further justifies the interpretation of $D_{1}$ with $D_{11}$ abovementioned. Moreover, for temperatures smaller than $280 \mathrm{~K}$ and larger than $350 \mathrm{~K}, D_{1}$, and thus, $D_{11}$ follows the temperature and pressure dependence often reported in the literature. It increases with increasing temperature [13, 33,34 ] and decreases with increasing pressure [33-35]. For temperatures between (280 and 350) K, $D_{1}$ assigned to be $D_{11}$ decreases with decreasing pressure due to the vicinity of the plait critical point $[36,37]$. The closer temperatures and pressures to the plait critical point, the more the critical slowing down is reflected by $D_{11}$.

\subsection{Diffusivities in the Supercritical State Along Isochores}

In the supercritical sate, we analyzed the diffusivities accessible by DLS along five isochores, including the critical isochore predicted by the GERG-2008 EoS [26]. The diffusivities are summarized as a function of the temperature difference $\left(T-T_{\mathrm{c}}\right)$ 
Fig. 6 Slow or Fick diffusivity as a function of the distance away from $T_{\mathrm{c}}$ along five isochores. The dashed line indicates a fit of the data along the critical isochore by a power law
- $\rho_{\mathrm{c}}=239.61 \mathrm{~kg} \cdot \mathrm{m}^{-3}$
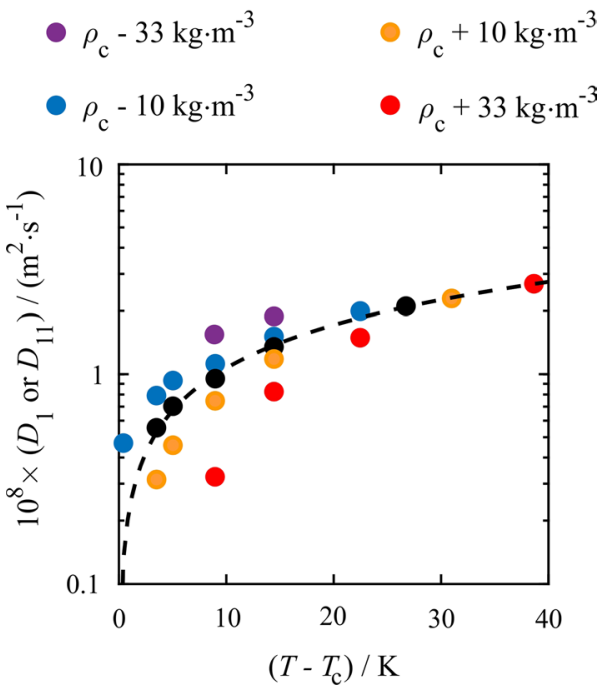

in Fig. 6. In most cases, only one hydrodynamic mode could be resolved in the CFs. According to the previous discussion, the single diffusivity can be associated with $D_{11}$. As shown in Fig. 6, this is confirmed by its behavior which follows the trend expected for $D_{11}$ which slows down approaching $T_{\mathrm{c}}$. Asymptotically close to the plait critical point of a binary mixture, the divergence of the correlation length of the fluctuations is reflected by the critical slowing down of the Fick diffusion coefficient. In the near-critical region, along the critical isochore, $D_{11}$ vanishes like the inverse of the correlation length to the power of 0.67 [36] and its behavior is the sum of a background and a vanishing contribution [4]. For the diffusivities obtained along the predicted critical isochore, a fit in form of a power law is indicated by the dashed line in Fig. 6. Here, the exponent was found to be $0.68 \pm 0.06$ which is in agreement with literature [38]. Because of the small amount of data points along the critical isochore and the lack of measurements in the close vicinity of the critical temperature, however, a data correlation which captures the background contribution was not possible. Far away from the plait critical point, our DLS measurements show that $D_{11}$ vary slowly with temperature and density. In the near-critical region, $D_{11}$ is decreasing with increasing density.

\subsection{Diffusivities in the Gas State Along Isotherms}

To explore the accessibility of diffusivities by DLS in the gas state, we analyzed the mixture at different pressures for temperatures of $(293,303,323$ and 353) $\mathrm{K}$. For all these states, only one hydrodynamic mode was resolvable in the CFs from which a mixed diffusivity $D_{\mathrm{m}}$ is derived. It was found to be decreasing with increasing pressure and decreasing temperature. The uncertainty of $D_{\mathrm{m}}$ is considerably larger at low densities in comparison to the high-density region. This is the 
result of a weaker amplitude of the signals associated with fluctuations in temperature and concentration in the low-density region, where optical contrast factors and other thermophysical properties are comparatively small. The pressure dependence of $D_{\mathrm{m}}$ obtained along these isotherms together with its experimental expanded uncertainty is displayed in Fig. 7a. Figure $7 \mathrm{~b}$ shows the behavior of the predicted Rayleigh ratio in the gas state for pressures up to $8 \mathrm{MPa}$. $\mathfrak{R}$ is slightly larger than unity far from the dew point line and increases with increasing pressure and reducing temperature. In the same region, the Lewis number is expected to be close to unity and the trend of the mixed diffusivity $D_{\mathrm{m}}$ agrees with the behavior of $a$ and $D_{11}$ [34]. It should be mentioned that the uncertainty for the theoretical $\mathfrak{R}$ values is large in the gas phase. Therefore, the single mode resolved in the CFs determined in this region can neither be associated with fluctuations in temperature nor in concentration, and thus, the single diffusivity is interpreted with a mixed diffusivity. The interpretation is affirmed by a comparison with Fick diffusivity data from the literature. $D_{\mathrm{m}}$ is $27 \%$ smaller than the Fick diffusivity measured by Zangi et al. [39] at $T=293.10 \mathrm{~K}$ and $p=0.500 \mathrm{MPa}$. In accordance with the trend of the predicted $\mathfrak{R}$ values from the gas state, diffusivities $D_{\mathrm{m}}$ obtained at low pressures $(p<1 \mathrm{MPa})$ are closer to $a$, while the ones obtained at higher pressures $(p>7 \mathrm{MPa})$ are closer to or matching with $D_{11}$.

Fig. 7 (a) Mixed diffusivity $D_{\mathrm{m}}$ obtained along different isotherms in the gas state. (b) Isolines of the Rayleigh ratio predicted with the GERG-2008 EoS [26] in the gas state
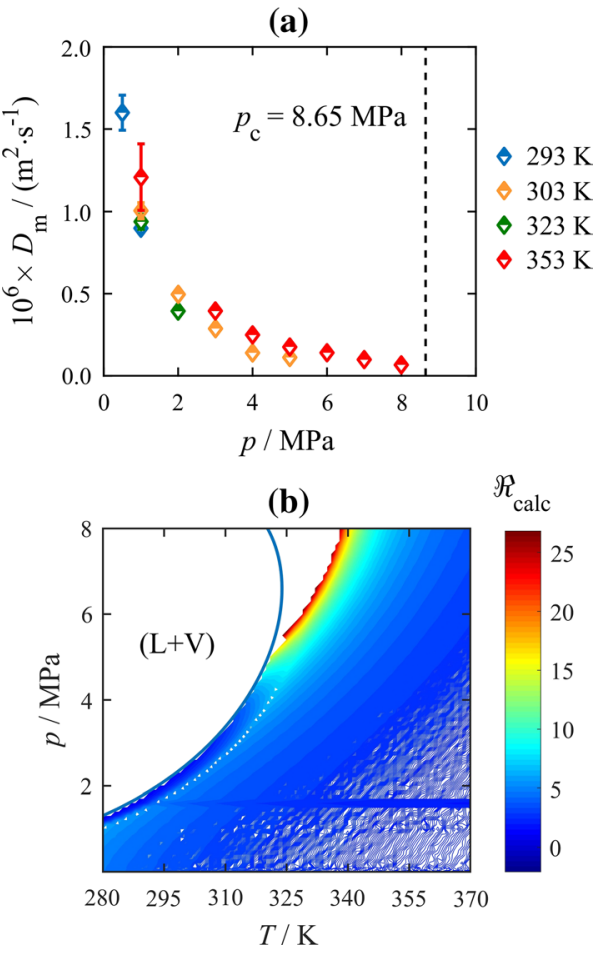


\subsection{Diffusivities in the Two-Phase Region Along Isotherms}

Depending on the volume fraction of liquid to vapor, the diffusivities in the twophase region were determined either in both the saturated liquid and vapor phases or only in one of them. While keeping the global composition of the mixture in the sample cell including the pressurizing system constant, the composition in each phase varies with pressure and temperature at vapor-liquid equilibrium. The mole fraction of propane in the saturated liquid and vapor phase was estimated with the GERG-2008 EoS [26] using measured temperatures and pressures. Two hydrodynamic modes were present in the experimental CFs obtained in the saturated liquid phase, allowing the simultaneous determination of $D_{1}$ and $D_{2}$, except at $T=323.04 \mathrm{~K}$ and $p=8.000 \mathrm{MPa}$. For this state, it should be mentioned that in the experiment the sample was found to be in the two-phase region while, according to the GERG-2008 EoS [26], it should exist in the gas state. In the saturated vapor phase, only one

Fig. 8 Pressure dependence of the slow or Fick diffusivity (top) and of the fast or thermal diffusivity (middle) in the saturated liquid phase at vapor-liquid equilibrium (2P-L) as well as in the liquid (L) or supercritical (SC) state for temperatures of (293, 303 and 323) K. Evolution of the corresponding Rayleigh ratio as a function of pressure (bottom)

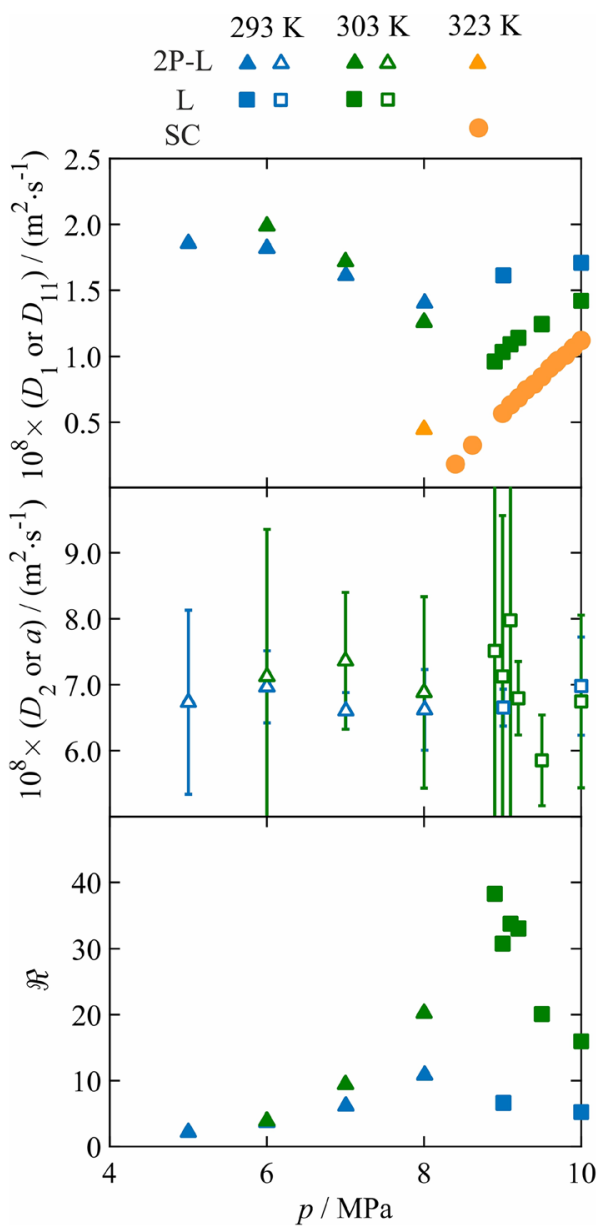


mode was accessible for all temperatures and pressures. Owing to the methodology used in this work, it was not possible to determine theoretically $\mathfrak{R}$ values within the two-phase region.

Figure 8 shows the diffusivities and Rayleigh ratios as function of pressure measured by DLS in the saturated liquid phase (2P-L) at $T=(293,323$ and 353) K. For the same temperatures, some results obtained in the liquid (L) and supercritical (SC) states are also depicted in Fig. 8. As shown in the upper and middle parts of Fig. 8, while $D_{1}$ or $D_{11}$ decreases with increasing pressure within the two-phase region and increases in the liquid and supercritical states, $D_{2}$ or $a$ seems to be independent on pressure and temperature. The larger uncertainties for $D_{2}$ or $a$ are due to the smaller amplitude of the corresponding thermal mode $(\mathfrak{R}>1)$, see lower part of Fig. 8. Because the diffusivity ratio $D_{2} / D_{1}$ is much larger than 2 , any mode coupling is assumed to be absent. Together with $\Re>1$, this justifies that $D_{1}$ and $D_{2}$ can be interpreted to be $D_{11}$ and $a$, respectively. A clear discontinuity can be found for $D_{11}$ between the two-phase region and the liquid or supercritical state which is most probably related to the change in the mixture composition. The closer the temperature to $T_{\mathrm{c}}$, the more pronounced the curvature connected with the description of $D_{11}$ as a function of pressure. In the saturated liquid phase, the Rayleigh ratio increases between the dew and bubble points. In the liquid state, it decreases with increasing pressure. Also for the Rayleigh ratio, the closer the temperature to $T_{\mathrm{c}}$, the more pronounced is its change by a variation in pressure around the bubble point.

The pressure dependence of the single diffusivity obtained by DLS in the saturated vapor phase (2P-V) along the aforementioned isotherms is shown in Fig. 9. Here, besides the data in the two-phase region, some data for the gas state are included for comparison purposes. For all temperatures $T \geq 303 \mathrm{~K}$, an increasing pressure leads to a decreasing $D_{\mathrm{m}}$ value, while at $T=293 \mathrm{~K}$ an increase in $D_{\mathrm{m}}$ can be observed. $D_{\mathrm{m}}$ exhibits a continuous behavior while crossing the dew point line.

For all thermodynamic states investigated in this work, diffusivities and Rayleigh ratios are summarized in Table 1. Following the mode interpretation discussed in the previous sections, the diffusivities have been assigned to the thermal, Fick, or mixed diffusivity.

Fig. 9 Pressure dependence of the mixed diffusivity $D_{\mathrm{m}}$ in the saturated vapor phase at vaporliquid equilibrium (2P-V) and in the gas state $(\mathrm{G})$ for temperatures of $(293,303$ and 323) K

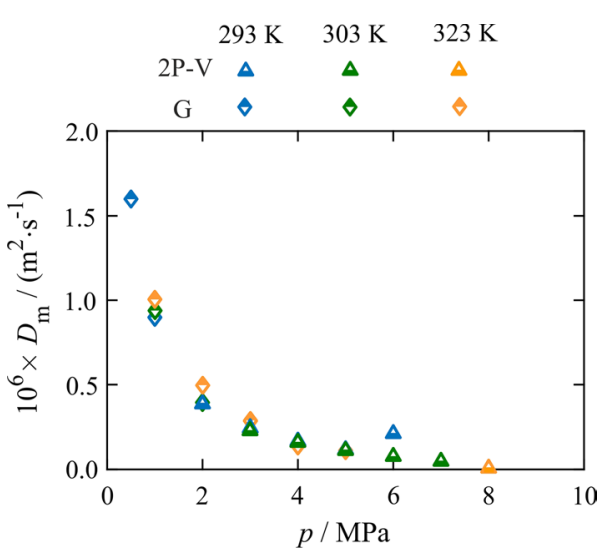




\section{Conclusion}

For an equimolar methane-propane mixture, DLS experiments were performed at temperatures between (283 and 363) $\mathrm{K}$ and pressures between (0.5 and 12) MPa, comprising the gas, liquid, and supercritical state as well as the two-phase region. Two hydrodynamic modes were resolved in the liquid state and saturated liquid phase for densities larger than 1.15 times the critical density. Here, with the help of theoretical and experimental Rayleigh ratios, it could be shown that the slow mode is always associated with the Fick diffusivity and the fast mode with the thermal diffusivity. For all other thermodynamic states, only one hydrodynamic mode was determined. While in the supercritical state the single mode is associated with the Fick diffusivity, in the gas state it is associated with a mixed diffusivity. This is because both the Rayleigh ratio and the Lewis number are close to unity in the gas state. The lower the density, the closer the mixed diffusivity to the thermal diffusivity. In the other case, the mixed diffusivity comes close to the Fick diffusivity. For the equimolar methane-propane mixture, a coupling between heat and mass transfer is assumed to be negligible over the complete range of temperatures and pressures investigated in this work. The diffusivities show the general temperature and pressure behavior reported in the literature, including the slowing down of the Fick diffusivity approaching the vicinity of the plait critical point. The average expanded uncertainty $(k=2)$ for the Fick, thermal, and mixed diffusivity as well as for the Rayleigh ratio are $(1.6,20.8,4.8$ and 27.1) \%, respectively. These first experimental data for the Fick, thermal, or mixed diffusivity as well as for the Rayleigh ratio of an equimolar methane-propane mixture across its two-phase region may further stimulate theoretical and experimental work in connection with a fundamental understanding of transport and other thermophysical properties.

Acknowledgements Open Access funding provided by Projekt DEAL. This work was supported financially by the German Research Foundation (Deutsche Forschungsgemeinschaft, DFG) by the research Grant DFG FR 1709/12-1.

\section{Compliance with Ethical Standards}

Conflict of interest The authors declare no competing financial interest.

Open Access This article is licensed under a Creative Commons Attribution 4.0 International License, which permits use, sharing, adaptation, distribution and reproduction in any medium or format, as long as you give appropriate credit to the original author(s) and the source, provide a link to the Creative Commons licence, and indicate if changes were made. The images or other third party material in this article are included in the article's Creative Commons licence, unless indicated otherwise in a credit line to the material. If material is not included in the article's Creative Commons licence and your intended use is not permitted by statutory regulation or exceeds the permitted use, you will need to obtain permission directly from the copyright holder. To view a copy of this licence, visit http://creativecommons.org/licen ses/by/4.0/. 


\section{References}

1. B.J. Berne, R. Pecora, Dynamic Light Scattering: With Applications to Chemistry, Biology, and Physics (Dover Publ (Courier Corporation), Mineola, 2000)

2. P. Fröba, Dynamic Light Scattering (DLS) for the Characterization of Working Fluids in Chemical and Energy Engineering, Friedrich-Alexander-Universität, Habil. Thesis, (2009).

3. M.A. Anisimov, V.A. Agayan, A.A. Povodyrev, J.V. Sengers, E.E. Gorodetskii, Phys. Rev. E 57, 1946 (1998)

4. A.P. Fröba, S. Will, A. Leipertz, Int. J. Thermophys. 21, 603 (2000)

5. B.J. Ackerson, H.J.M. Hanley, J. Chem. Phys. 73, 3568 (1980)

6. L.D. Landau, E.M. Lifshitz, Statistical Physics, vol. 5 (Pergamon, London, 1959)

7. J.M. Ortiz de Zárate, J.V. Sengers, Hydrodynamic Fluctuations (Elsevier, Amsterdam, 2006)

8. D.A. Ivanov, T. Grossmann, J. Winkelmann, Fluid Phase Equilib. 228-229, 283 (2005)

9. D.A. Ivanov, J. Winkelmann, Int. J. Thermophys. 29, 1921 (2007)

10. C. Giraudet, T. Klein, G. Zhao, M.H. Rausch, T.M. Koller, A.P. Fröba, J. Phys. Chem. B 122, 3163 (2018)

11. A. Heller, M.S.H. Fleys, J. Chen, G.P. Van Der Laan, M.H. Rausch, A.P. Fröba, J. Chem. Eng. Data 61, 1333 (2016)

12. A. Heller, T.M. Koller, M.H. Rausch, M.S.H. Fleys, A.N.R. Bos, G.P. van der Laan, Z.A. Makrodimitri, I.G. Economou, A.P. Fröba, J. Phys. Chem. B 118, 3981 (2014)

13. M. Piszko, W. Wu, S. Will, M.H. Rausch, C. Giraudet, A.P. Fröba, Fuel 242, 562 (2019)

14. R.B. Eldridge, Ind. Eng. Chem. Res. 32, 2208 (1993)

15. F. Montel, J. Bickert, and M. Royer, in SPE 85668 (2003)

16. T. Huthwelker, T. Peter, J. Chem. Phys. 105, 1661 (1996)

17. U.A. Higgoda, R. Hellmann, T.M. Koller, A.P. Fröba, Fluid Phase Equilib. 481, 15 (2019)

18. J.P. Boon, S. Yip, Molecular hydrodynamics (Dover Publ (Courier Corporation), Mineola, 1980)

19. P.N. Segrè, J.V. Sengers, Phys. A 198, 46 (1993)

20. H.A. Lorentz, The Theory of Electrons and Its Applications to the Phenomena of Light and Radiant Heat, 2nd edn. (B.G. Teubner, Leipzig, 1916)

21. W.B. Li, R.W.G. Segré, J.V. Sengers, M. Lamvik, J. Chem. Phys. 101, 5058 (1994)

22. M. Gebhardt, W. Köhler, A. Mialdun, V. Yasnou, V. Shevtsova, J. Chem. Phys. 138, 114503 (2013)

23. C. Giraudet, L. Marlin, D. Bégué, F. Croccolo, H. Bataller, J. Chem. Phys. 144, 134304 (2016)

24. M. Piszko, K. Batz, M.H. Rausch, C. Giraudet, A.P. Fröba, J. Chem. Eng. Data 65, 1068 (2020)

25. M.R. Moldover, J.V. Sengers, R.W. Gammon, R.J. Hocken, Rev. Mod. Phys. 51, 79 (1979)

26. O. Kunz, W. Wagner, J. Chem. Eng. Data 57, 3032 (2012)

27. E. W. Lemmon, I. H. Bell, M. L. Huber, and M. O. McLinden, (2018)

28. K.N. Seneviratne, T.J. Hughes, M.L. Johns, K.N. Marsh, E.F. May, J. Chem. Thermodyn. 111, 173 (2017)

29. A.M. Abudour, S.A. Mohammad, R.L. Robinson, K.A.M. Gasem, Fluid Phase Equilib. 349, 37 (2013)

30. NIST Computational Chemistry Comparison and Benchmark Database NIST Standard Reference Database Number 101 Release 19, April 2018, Editor: Russell D. Johnson III http://cccbdb.nist. gov/, (n.d.)

31. M. Richter, M.O. McLinden, J. Chem. Eng. Data 59, 4151 (2014)

32. M. A. Anisimov, E. E. Gorodetskii, V. D. Kulikov, and J.V. Sengers, Phys. Rev. E 51, (1995)

33. I. Lizarraga, C. Giraudet, F. Croccolo, M. Bou-ali, H. Bataller, Microgravity Sci. Technol. 28, 545 (2016)

34. I. Medina, J. Chromatogr. A 1250, 124 (2012)

35. C. Giraudet, H. Bataller, F. Croccolo, Eur. Phys. J. E 37, 1 (2014)

36. R.F. Chang, P.H. Keyes, J.V. Sengers, C.O. Alley, Phys Rev Lett. 27, 1706 (1970)

37. J. Luettmer-Strathmann, J.V. Sengers, Int. J. Thermophys. 15, 1241 (1994)

38. J.V. Sengers, J.G. Shanks, J. Stat. Phys. 137, 857 (2009)

39. P. Zangi, M.H. Rausch, A.P. Fröba, Int. J. Thermophys. 40, 18 (2019)

Publisher's Note Springer Nature remains neutral with regard to jurisdictional claims in published maps and institutional affiliations. 